\title{
BREVES RESEÑAS BIOGRÁFICAS DE LOS AUTORES
}

\section{Carlos Aguilar Blanc}

Doctor cum laude en Pensamiento Político, Democracia y Ciudadanía, por la Universidad Pablo de Olavide de Sevilla. Ha impartido docencia en varios programas de Master en la Universidad Internacional de Andalucía y en estudios de grado en la Universidad Pablo de Olavide de Sevilla. Actualmente ejerce como profesor de Filosofía del Derecho y valores democráticos, género y paz en la Universidad de Huelva. Miembro activo del Grupo de Investigación SEJ-277 "Derechos Fundamentales: Teoría general", en cuyo marco institucional desarrolla su actividad investigadora. Es autor de diversos trabajos en materia de Derechos Humanos, Filosofía Política y Filosofía del Derecho. Licenciado en Derecho, ha sido abogado en ejercicio durante varios años en diversos bufetes de ámbito nacional e internacional. Tiene un Master en Estudios Latinoamericanos (UNIA). Ha realizado varias visitas como profesor de grado y postgrado así como en calidad de investigador y conferenciante en algunas universidades brasileñas. Su actividad investigadora se ha centrado principalmente en el estudio de los fundamentos teóricos y jurídicos del Terror de Estado, abordando así mismo la conflictividad existente entre la seguridad pública, la amenaza terrorista y los derechos humanos.

\section{Jorge Francisco Aquirre Sala}

Doctor en Filosofía por la Universidad Iberoamericana de Ciudad de México. Actualmente afiliado a la Universidad Autónoma de Nuevo León donde es líder del Cuerpo Académico "Democracia y Sustentabilidad". Cuenta con de más de 200 citas, por autores y tesistas, a sus artículos y libros publicados en México, Chile, Argentina, Uruguay, Brasil, Colombia, España, Alemania, Austria y EE.UU. Entre sus publicaciones destaca: La democracia líquida. Los nuevos modelos políticos en la era digital, Barcelona: UOC. "Entre las redes sociales y los medios tradicionales: preferencias del electorado neoleonés ¿Benditas redes sociales?" en Gestión y formación audiovisual para crear contenidos en redes sociales. Madrid: McGraw Hill. ID Orcid: 0000-0002-5805-408

\section{Verna Alcalde González}

Licenciado en Comunicación Audiovisual y Máster en Análisis Sociocultural del Conocimiento y de la Comunicación por la Universidad Complutense de Madrid y Graduado en Filosofía por la UNED. Actualmente trabaja como investigador predoctoral en la Universitat Oberta de Catalunya. Su proyecto de tesis toma como objeto de estudio la acción sindical y política de las kellys para abordar el tema de la acción colectiva en un contexto sociolaboral caracterizado por la precarización de las condiciones de trabajo, la crisis del movimiento sindical y el ciclo de protestas feministas incardinado en la denominada cuarta ola del feminismo. 


\section{Jordi Arcarons Bullich}

Catedrático de Economía Aplicada. Profesor de Econometría de la Empresa en el Grado de Administración y Dirección de Empresas de la Facultad de Economía y Empresa de la Universidad de Barcelona. Ha publicado diversos manuales de Econometría. Ha desarrollado y elaborado diversos micro simuladores de reformas fiscales y de cálculo de beneficios fiscales que actualmente utiliza la Generalitat de Catalunya y el Gobierno de Canarias. Es autor de diversos modelos de micro simulación para analizar el impacto de la implantación de una Renta Básica. Es coautor del libro Renta básica incondicional. Una propuesta de financiación racional y justa (Serbal, 2017). Ha publicado diversos artículos de análisis sobre la Renta Básica. Es miembro de la Red Renta Básica (www.redrentabasica.org).

\section{Juan Manuel Belmonte Lozano}

Licenciado en Ciencias Políticas y de la Administración por la Universidad de Granada (2011); posee un Máster en Derecho Constitucional por la Universidad de Sevilla (2014), otro en Historia de Europa, el Mundo Mediterráneo y su difusión Atlántica por la Universidad Pablo de Olavide (2015); y un título de Posgrado en Cooperación Internacional para el Desarrollo por la Universitat Oberta de Catalunya (2019). Ha publicado la monografía $E /$ derecho indígena a la tierra en México, México, REUS, 2016; y ha realizado varias estancias académicas y de investigación: Universidad Autónoma de Tamaulipas, Université Libre de Bruxelles y Universidad Autónoma del Estado de Morelos.

\section{Emmanuel Biset}

Doctor en Filosofía por la Université Paris 8 (Francia) y por la Universidad Nacional de Córdoba (Argentina). Posdoctorado por la Universitá Degli Studi di Padova. Investigador del CONICET y Profesor de la Universidad Nacional de Córdoba (Argentina). Es docente y miembro del Comité Académico del Doctorado en Ciencia Política de la UNC. Director del Programa de Investigación en Teoría Política del CIECS de la UNC y el CONICET. Ha publicado los libros «Violencia, justicia y política», «El Signo y la Hiedra. Escritos sobre Jacques Derrida» y «El ritmo y la distancia». Ha compilado, entre otros, los libros: «Soberanías en deconstrucción», «Sujeto. Una categoría en disputa», «Derrida político» y «Ontologías políticas».

\section{David Casassas Marqués}

Profesor de teoría social y política en la Universidad de Barcelona, donde trabaja en el seno del Grup de Recerca en Ėtica economicosocial i Epistemologia de les Ciències Socials (GREECS). Ha sido investigador en la Universidad Católica de Lovaina, en la Universidad de Oxford y en la Universidad Autónoma de Barcelona. Ha sido Secretario de la Basic Income Earth Network (BIEN) y forma parte del Consejo Asesor Internacional de dicha organización. Es Vicepresidente de la Red Renta Básica y miembro del Consejo de Redacción de la revista SinPermiso. Colabora también con el Observatorio de los Derechos Económicos, Sociales y Culturales (DESC). Su último libro es Libertad incondicional. La renta básica en la revolución democrática (Paidós, 2018). 


\section{Marta Consuegra-Fernández}

Doctora en Biomedicina por la Universitat de Barcelona (UB) y máster en Comunicación especializada por la misma universidad. Profesora de grado y máster de comunicación de la salud y científica en la Facultat de Filologia i Comunicació de la Universidad de Barcelona. Investigadora colaboradora del Observatori de Bioètica i Dret de la UB y grupos de investigación del Hospital Clínic de Barcelona y del Consorci Corporació Sanitària Parc Taulí de Sabadell. Colaboradora de las revistas de divulgación científica "Investigación y Ciencia" y "Mente y Cerebro" de la editorial Prensa Científica y documentalista del programa de ciencia de TVE "El cazador de Cerebros" de la productora Minifilms.

\section{Itziar de Lecuona}

Doctora en Derecho (línea de Investigación en Bioética y Derecho) y Master en Bioética y Derecho por la Universidad de Barcelona; Profesora Agregada del Departamento de Medicina y Subdirectora del Observatorio de Bioética y Derecho (OBD) - Cátedra UNESCO de Bioética de la Universidad de Barcelona. Miembro del Grupo de Investigación Consolidado «Bioética, Derecho y Sociedad» de la Generalitat de Catalunya. Miembro del Grupo de Trabajo Multidisciplinar sobre la Covid-19 del Ministerio de Ciencia e Innovación. Miembro del Comité de Bioética de Cataluña (2015-2020); del Comité de Ética en Investigación del Hospital Clínico de Barcelona (2012-2020); de la Comisión de Bioética de la Universidad de Barcelona y del Comité de Ética de la Universidad Politécnica de Cataluña. Sus líneas de investigación preferentes son: los aspectos bioéticos, jurídicos y sociales de la investigación en seres humanos, los comités de ética en investigación, la investigación con datos masivos y la protección de la intimidad. Ha participado y participa en proyectos de investigación competitivos autonómicos, nacionales e internacionales. Conferenciante en congresos internacionales de reconocido prestigio en bioética, ha publicado libros y artículos en revistas científicas indexadas en los principales repositorios de calidad reconocida. Ha sido investigadora visitante de la Universidad de Bologna, de UNESCO Paris sección de Bioética y del Instituto de Salud Carlos III, Madríd. Consultar la Actividad investigadora y producción científica de Itziar de Lecuona, Portal de la Recerca de Catalunya.

\section{Jad El Khannoussi}

Doctor por la Universidad de Cádiz. Investigador y lector en el Departamento árabe de la misma universidad. Conferenciante y articulista en revistas científicas españolas e internacionales. Sus estudios abarcan diversas temáticas. Entre sus publicaciones destacan las siguientes: Libro: El Khannoussi, J.: El mundo árabe en la Posguerra Fría: Un dilema geopolítico complejo. Cádiz: Book, 2019, Capítulos de libros: El Cine egipcio y la propaganda del poder: Egipto de Nasser a Mubarak, Leadder to Damascus: el Amor sobre la sinfonías revolucionarias. Artículos: Political Islam: Beyond the End Or Post Islam, Los factores externos e internos de la revolución Siria, El gran Oriente Medio, La primavera Árabe: ¿oportunidad o desafío?, Turkey and its geopolitical reserves: Between Est and West, Iran and the Gula status: statics and Dynamics, El mundo árabe y la democracia ¿Un matrimonio pendiente?, Rusia and The Horn of Africa: A quiet 
Return?, Crimea ¿Un motivo de Guerra?, Ucrania: entre viejas y nuevas potencias, Irak iun dilema complejo?, Oriente Medio ¿el caos?, Afrin: ¿el verdadero examen para Turquía?, Irán entre la ira interior y la presión exterior, Idlib: Un acuerdo sobre un cielo caliente, The Strait of Malacca: a Geoestrategic enigma for China, Turquía: hacia una nueva postura exterior, Manantial de Paz: ¿Una cuestión de supervivencia para Ankara?, Jasta ¿el principio del divorcio entre Washington y Riyad?, Palestina ¿Una causa Humana?, Jerusalen: siempre será multicultural, Declaración de Balfour: ¿Una carta de dimensiones estratégicas?, El referndúm de Kurdistán:¿derecho o inestabilidad?, Unión europea: ¿Reformas o desintegración?, Los principales impactos sobre el pensamiento europeo ¿Renacimiento?, El pensamiento político de Ibn Jaldún: Términos esenciales,

\section{René Fernando Estévez Abad}

Doctor en Medicina, Especialista en neurología, Magister en Bioética, PhD por la Universidad de Barcelona. Tutor del Máster de Bioética y Derecho de la UB, Profesor Titular de la Facultad de Ciencias Médicas en la Universidad de Cuenca en las cátedras de neurofisiología y bioética. Miembro del Consejo Académico de la Universidad de Cuenca. Ex director de posgrados de medicina de la Universidad de Cuenca. Ex Presidente y Vicepresidente del Colegio de Médicos del Azuay. Investigador activo del proyecto de Neurociencias, Neuropsicología y daño cognitivo de la Universidad del Azuay. Investigador en proyectos de estudio sobre: envejecimiento saludable, demencia y soporte cognitivo, problemáticas de la bioética y neurociencia, neuroética y educación médica.

\section{Fernando Filqueira}

Licenciado en Sociología por la Universidad de La República y Doctor en Sociología por la Nothwestern University. Ha publicado libros y artículos en revistas especializadas de políticas sociales, estructura social, políticas públicas, educación y desarrollo de América Latina. Fue coordinador del Programa de Estudios y Estadísticas Educativas del sistema educativo público en Uruguay. Fue director del Aréa de Gestión y Evaluación del Estado de la Oficina de Planeamiento y Presupuesto de Uruguay y oficial de asuntos sociales en la CEPAL en Chile. Integra como lead author el Panel Internacional para el Progreso Social liderado por Amartya Sen. En la actualidad es profesor e investigador de la Facultad de Ciencias Sociales de la Universidad de la República y Jefe de oficina del Fondo de Población de Naciones Unidas en Uruguay.

\section{Carmen García Pérez}

Abogada y economista. Licenciada en Derecho y Economía por la Universidad Carlos III de Madrid, y Máster en Dirección, Gestión e Intervención en Servicios Sociales por la Universidad Ramón Llull de Barcelona. Es socia de un despacho profesional especializado en la consultoría y abogacía en el sector público. Entre otras cosas, es abogada de un centro de servicios sociales. Por su formación y profesión cuenta con una visión transversal de las políticas públicas sociales, que analiza desde diferentes perspectivas. Cuenta con publicaciones sobre Renta Básica, en las que se analiza su conveniencia y viabilidad. Más información en www.cgarciaperez.me 


\section{Eduardo Garzón Espinosa}

Licenciado en Economía y Administración y Dirección de Empresas por la Universidad de Málaga, máster en Economía Internacional por la Universidad Complutense de Madrid, doctorando por la Universidad Autónoma de Madrid, profesor ayudante en el departamento de Economía y Hacienda Pública de la Universidad Autónoma de Madrid. Autor y coautor de varios artículos publicados en revistas de economía de impacto y de varios libros y capítulos de libros. Autor de diversas comunicaciones en distintos congresos nacionales e internacionales de economía. Organizador de diversas jornadas de economía en la Universidad Complutense de Madrid y en la Universidad de Málaga. Colaborador asiduo de programas de televisión y de radio para hablar como experto en economía. Con blog personal y cuentas en redes sociales con más de 100.000 seguidores en total. También autor de artículos de economía en diarios nacionales.

\section{Rubén M. Lo Vuolo}

Economista, egresado de la Universidad Nacional del Litoral (Argentina) y la University of Pittsburgh (EEUU). Actualmente es Investigador del Centro Interdisciplinario para el Estudio de Políticas Públicas (Buenos Aires, Argentina). Ha sido investigador visitante en universidades del extranjero y consultor de diversos organismos internacionales. Publicó libros y artículos en revistas especializadas sobre temas vinculados con políticas sociales, mercado de empleo, teoría y política económica. Ha sido investigador visitante en universidades del extranjero y consultor de diversos organismos internacionales. Su último libro es Políticas Públicas y Democracia en Argenti- na. Crónicas de un País que no Aprende (2017). Es Presidente de la Red Argentina de Ingreso Ciudadano (Redaic).

\section{Manuel Jesús López Baroni}

Licenciado en derecho (Universidad de Sevilla) y doctor en derecho (Universidad de Barcelona); también licenciado y doctor en filosofía (UNED). Cursó el título de experto universitario en criminología en el Instituto Andaluz Interuniversitario de Criminología (tres cursos académicos) y el Máster en Pensamiento Político en la Universidad Pablo de Olavide, de Sevilla. Es profesor de filosofía del derecho y política en la Universidad Pablo de Olavide, de Sevilla, así como miembro del Observatorio de Bioética y Derecho de la Universidad de Barcelona. Ha centrado sus investigaciones en los últimos años en bioética, en concreto, en las implicaciones legales y éticas de las denominadas tecnologías disruptivas (biotecnología, biología sintética, nanotecnología, Inteligencia Artificial y neurotecnología). Forma parte de los programas de doctorado en derecho y bioética de la Universidad de Barcelona, y de biotecnología e ingeniería genética, de la Universidad Pablo de Olavide. Ha sido o es profesor del Máster en Bioética y Derecho de la Universidad de Barcelona y del Master in Manufacturing of Advanced Therapy Medicinal Products, de la Universidad de Granada y del Instituto Andaluz de Terapias Avanzadas. Participa en un proyecto del Ministerio para reformar el Convenio europeo de bioética y en un Libro Blanco de la Nanotecnología en Cataluña.

\section{Isabel Victoria Lucena Cid}

Licenciada en Filosofía por la Universidad de Sevilla, Doctora con mención Europea 
y profesora del Área de Filosofía del Derecho y Política de la Universidad desde 2001. Premio Doctorado 2008. Es autora de múltiples trabajos relacionados con los Derechos Humanos, la Filosofía del Derecho y Política. En materia de Cooperación Internacional al Desarrollo ha realizado diversas investigaciones y organizado e impartido docencia en cursos de especialización y Máster sobre la materia. Ha realizado diversas estancias de investigación en universidades del Reino Unido, Institut for Development Studies de la Universidad de Sussex (2003 y 2014) y el Oxford Centre for Ethics and Philosophy of Law de la Universidad de Oxford (2005, 2015, 2016 y 2017). Ha sido Visiting Fellow en el Centre Global Governance en la London School of Economics and Political Science (2010, en el marco del programa Castillejo). En Italia ha desarrollado distintos trabajos de investigación en la Universidad de Pavía, Universidad de Milán (2004) y el Pontificio Istituto di Studi Arabi ed Islamistica de Roma (2008), así como en el L'Istituto di Teoria e Tecniche dell'Informazione Giuridica (ITTIG), del Consiglio Nazionale delle Ricerche, en Florencia (2013). En el ámbito de la gestión universitaria ha sido Vicedecana de Calidad, Estrategia y Nuevas Tecnologías de la Facultad de Derecho (2009-2013), Vicesecretaria General de la Universidad 2013-2016. Vicerrectora de Internacionalización 2016-2020.

\section{Noel Manzanares Blanco}

Profesor de Teoría Sociopolítica en la Universidad de Camagüey Ignacio Agramonte Loynaz, Cuba. Maestro en Estudios Sociales y Analista político. Columnista de $\mathrm{Cu}$ bainformación y Kaos en la Red, sitios digitales de España. Ha publicado en diversas revistas cubanas y extranjeras, y sus artículos han sido reproducidos en Latinoamérica, EEUU, Europa y África. Ha investigado/ investiga sobre la conducción del proceso revolucionario cubano, la política de Estados Unidos de América y la Izquierda en Latinoamérica -entre otros asuntos-. Preside la Cátedra Honorífica de Estudio del Pensamiento y Obra de Fidel Castro Ruz, en su institución. ORCID ID: https:// orcid.org/0000-0002-9472-2711.

\section{Julio Martínez-Cava Aquilar}

Investigador predoctoral y docente en el Departamento de Sociología de la Universidad de Barcelona, donde trabaja con el Grup de Recerca en Ėtica economicosocial i Epistemologia de les Ciències Socials (GREECS). Licenciado en Filosofía (Universidad Complutense de Madrid) y con un Máster en Historia Contemporánea (Universidad Complutense de Madrid), sus líneas de investigación giran en torno a la historia del pensamiento republicano y socialista, el marxismo británico, las teorías de clase social o la renta básica. Es miembro del Comité de Redacción de Sin Permiso. Colabora también con la cooperativa editorial Cultura 21. Ha traducido ensayos del inglés al castellano de autores destacados como Guy Standing (editorial Pasado y Presente) o Alex Gourevitch (editorial Capitán Swing). Sus últimas publicaciones destacadas son: Martínez-Cava, J. "Enemigo a las puertas. La libertad política y los principios fiduciarios en el socialismo británico", Daimon. Revista Internacional de Filosofía, 81, 2020, pp. 161-177; Martínez-Cava, J. "Introducción" en E. P. Thompson, Costumbres en común. Estudios sobre la cultura popular, Madrid: Capitán Swing, 2019, pp. 11-45. 


\section{Karina Johana Martínez Jiménez}

Graduada en Filosofía por la Universidad de Cartagena (Colombia). Magíster en Derechos Humanos, Interculturalidad y Desarrollo, por la Universidad Pablo de Olavide (Sevilla), con el trabajo de fin de Máster: Los crímenes contra la humanidad en el caso colombiano: De la Unión Patriótica a los líderes sociales en el post-acuerdo. Miembro del grupo de investigación Civitas, de la Universidad de Cartagena. Colaboradora de revista Derecho Global. Experiencia de trabajo con población vulnerable y en trabajo voluntario con población migrante en organización humanitaria internacional. Orcid: 0000-0002-3593-241X

\section{Fabiana Parra}

Doctora, Licenciada y Profesora en Filosofía por la Universidad Nacional de La Plata (UNLP-Argentina), Magíster en Filosofía por la Universidad de Quilmes (UNQui). Investigadora en el Instituto de Investigaciones en Humanidades y Ciencias Sociales (IdIHCS) - Consejo Nacional de Investigaciones en Ciencia y Técnica CONICET - UNLP, Argenina. Profesora de Filosofía en la Universidad Nacional de La Plata (UNLP). Directora del Proyecto de extensión "Cuestionarlo todo para retejernos de otro modo" (UNLP). Integrante de proyectos de investigación sobre filosofía contemporánea y feminismos. Actualmente realiza su investigación posdoctoral financiada por CONICET: "Ideología y género. Lectura sintomal y genealogía conceptual" en el Centro de Investigaciones (CIEFI)- IdIHCS. Ha sido profesora invitada en la UNAM y en la UMSNH (México), en UNICAMP y UFRJ (Brasil), en Uchile y en la UGR (España).
Y ha realizado numerosas presentaciones y publicaciones en torno a la articulación entre ideología, género, crítica política, genealogías feministas e interseccionalidad. Integra la Colectiva de Filósofas Feministas de la UNLP.

\section{Daniel Raventós Pañella}

Doctor en Ciencias Económicas, Profesor Titular del Departamento de Sociología en la Facultad de Economía y Empresa de la Universidad de Barcelona, y miembro del grupo de investigación GREECS. Ha sido conferenciante invitado en universidades europeas y americanas, y uno de los introductores académicos de la propuesta de la renta básica. Fue uno de los fundadores de la Red Renta Básica en 2001, siendo desde entonces su presidente. $\mathrm{Ha}$ escrito diversos trabajos sobre teoría normativa republicana. Escribe regularmente en la revista editada en Estados Unidos Counterpunch. Parte de su actividad la dedica a la promoción pública de la renta básica, así como a la edición y organización de la revista Sin Permiso. Es coautor del libro Renta básica incondicional. Una propuesta de financiación racional y justa (Serbal, 2017). Ha colaborado en diversos libros editados en Gran Bretaña, Argentina, Turquía, Uruguay, Italia, Colombia, Estados Unidos, España y México. Ha publicado varios libros sobre distintos aspectos normativos y técnicos de la renta básica.

\section{Jesús Rodríguez Rojo}

Graduado en Ciencias Políticas y de la Administración por la Universidad Pablo de Olavide, Graduado en Sociología por la Universidad Pablo de Olavide, Máster universitario en Derechos Humanos, Intercul- 
turalidad y Desarrollo por la Universidad Pablo de Olavide. Diplomado en «Análisis crítico del capitalismo (El método marxista y su aplicación al estudio de la economía mundial)» por la Universidad Complutense de Madrid. Diplomado en "Geopolítica y Defensa Latinoamericana» por el Programa Latinoamericano de Educación a Distancia de la Universidad de Buenos Aires. Evaluador regular de artículos científicos. Director y conductor del programa radiofónico «Hablamos en Red», emitido en Radiópolis (88.0 FM en Sevilla). Es autor de un libro, tres capítulos de libro y diez artículos de revistas científicas. Ha presentado numerosas comunicaciones a congresos y seminarios. Ha sido director en el seminario «Marx y El capital en el mundo contemporáneo». Ha participado en varios proyectos de investigación. Sus líneas temáticas giran en torno a la teoría marxista y los derechos humanos.

\section{José María Seco Martínez}

Licenciado en Derecho con Grado de Sobresaliente. Doctor en Derecho con Sobresaliente Cum Laude por la Universidad de Sevilla. Letrado de la Administración de Justicia. Profesor Titular de Filosofía del Derecho. Su historial investigador destaca por: (i) sus numerosas publicaciones en medios de difusión adecuados, esto es, de relevancia científica en sus campos de estudio y que siguen, a juicio del solicitante, una línea de investigación coherente y permanente; (ii) su participación en proyectos y/o contratos de investigación de excelencia y de concurrencia competitiva ( 3 proyectos I+D+I, 2 proyectos de Excelencia, RCC Harvard University, etc), tanto nacionales como internacionales; y (iii) por su contribución en numerosos congresos y seminarios, al- gunos de ellos internacionales en universidades de referencia internacional, como la Universidad de Harvard. En el ámbito de las publicaciones el solicitante tiene en su haber más de 40 artículos, muchos de los cuales ocupan una posición muy destacada en los registros bibliométricos o ranking de revistas, con fuertes niveles de impacto en su especialidad y referenciadas en las principales bases de datos del campo científico técnico de referencia. Libros completos (como autor, editor y coautor) sobre temas de investigación directamente relacionados con su campo de estudio. Numerosos (22) capítulos de libro en editoriales de prestigio nacional e internacional, tales como DYKINSON, TECNOS, MARCIAL PONS, WOLTERS KLUWERS, ICARIA, etc.

\section{Lluís Torrens Mèlich}

Economista, magister en análisis económico por UAB-IAE y PDG por IESE. Director de Innovación Social del Área de Derechos Sociales, Justicia Global, Feminismos y LGTBI del Ayuntamiento de Barcelona. Profesor asociado de diversas universidades.

Especialista en economía aplicada, en 2005 recibió el premio Catalunya de Economía de la Societat Catalana d'Economia. Es miembro del consejo editorial de la Revista Economía Industrial. Es coautor del libro Renta básica incondicional. Una propuesta de financiación racional y justa (Serbal, 2017). Ha publicado diversos artículos de análisis sobre la Renta Básica. Es miembro de la Red Renta Básica (www.redrentabasica.org). 